Series A

I. MATHEMATICA

530

\title{
REMARKS ON THE REGULARITY OF THE SOLUTIONS OF A LINEAR PARTIAL DIFFERENTIAL EQUATION WITH CONSTANT COEFFICIENTS
}

BY

VÄINÖ JALAVA 
Copyright (C) 1972 by

Academia Scientiarum Fennica

ISBN $951-41-0086-7$

Communicated 12 October 197: by Rolf Nevaxistsa 
1. Let us consider a linear partial differential equation

$$
L u=f \text {, }
$$

where the differential operator $L$ has constant coefficients. We assume that $f$ belongs to the Sobolev space $H^{p}(\Omega) \quad(p \geqq 0)$, where $\Omega$ is a bounded open domain in the Euclidean space $R^{n}$. Let $L^{\prime}$ be the formal adjoint of $L$ and write $M=L^{\prime} L(1-\Delta)^{p}$. With the help of $M$ we construct a distribution space $X^{-}$such that $L^{2}(\Omega) \subset X^{-} \subset D^{\prime}(\Omega)$. We shall show that if $u \in X^{-}$is a distribution solution of (1), it can be decomposed

$$
u=u_{0}+\vartheta,
$$

where $u_{0} \in H_{0}^{p+r}(\Omega) \quad(r \geqq 0)$ and $\vartheta \quad$ satisfies

$$
\left(M^{2}+1\right) \vartheta=0 \text {. }
$$

Thus the part $u_{0}$ is at least as regular as the right hand side $f$ of (1). Since the second part $\vartheta$ satisfies the homogeneous equation (2), its regularity depends only on the differential operator $L$. Consequently, the question about the regularity of a solution of the inhomogeneous problem (1) returns to the question about the regularity of a solution of the homogeneous problem (2). Furthermore, this result will be applied to prove that weak $L^{2}$-solutions of hypoelliptic equations can be approximated in $L^{2}(\Omega)$ by $C^{\infty}$-functions.

2. Let $\Omega$ be a bounded open domain in the Euclidean space $R^{n}$. For a multi-index $\varrho=\left(\varrho_{1}, \ldots, \varrho_{n}\right)$ we write $D^{\circ}=D_{1}^{\varrho_{1}} \ldots D_{n}^{\circ}$ where $D_{i}=\partial / \partial x_{i}$. If $p \geqq 0$ is an integer, we denote by $H^{p}(\Omega)$ the Sobolev space consisting of the complex valued functions whose distribution derivates of order $\leqq p$ belong to $L^{2}(\Omega)$. The space $H^{p}(\Omega)$ is a Hilbert space with the inner product

$$
(u, v)_{p}=\sum_{|\varrho| \leqq p} \frac{p !}{\varrho_{1} ! \ldots \varrho_{n} !(p-|\varrho|) !} \int_{\Omega} D^{\varrho} u \overline{D^{\varrho} v} d x
$$

$\left(u, v \in H^{p}(\Omega)\right)$. Let $H_{0}^{p}(\Omega)$ be the closure of $C_{0}^{\infty}(\Omega)$ in $H^{p}(\Omega)$. 
We consider a differential operator

$$
L=\sum_{|\varrho| \leqq m} a_{\varrho} D^{\circ}
$$

with constant coefficients. There is an integer $r \geqq 0$ such that for each $p \geqq 0$ one has

$$
\|L \varphi\|_{p} \geqq k_{p}\|\varphi\|_{p+r} \quad\left(\varphi \in C_{0}^{\infty}(\Omega)\right),
$$

where $k_{p}$ is a positive constant (cf. [1], p. 177). The formal adjoint of $L$ is

$$
L^{\prime}=\sum_{|\varrho| \leqq m}(-1)^{|\varsigma|} \overline{a_{o}} D^{o} .
$$

Let us choose an integer $p \geqq 0$. Writing

$$
S=1-\Delta=1-\sum_{i=1}^{n} D_{i}^{2}
$$

one has for $f \in H^{p}(\Omega)$ and $\varphi \in C_{0}^{\infty}(\Omega)$

$$
(f, \varphi)_{p}=\left(f, S^{p} \varphi\right)_{0} .
$$

We consider the formally self-adjoint differential operator

$$
M=L^{\prime} L S^{p} .
$$

For $\varphi \in C_{0}^{\infty}(\Omega)$ we get by (3)

$$
(\varphi, M \varphi)_{0}=\left(L \varphi, S^{p} L \varphi\right)_{0}=\|L \varphi\|_{p}^{2} \geqq k_{p}^{2}\|\varphi\|_{p+r}^{2} .
$$

Let us define on $C_{0}^{\infty}(\Omega)$ an inner product

$$
(\varphi, \psi)_{X}=(\varphi, M \psi)_{0} \quad\left(\varphi, \psi \in C_{0}^{\infty}(\Omega)\right) \text {. }
$$

We denote by $X$ the completion of $C_{0}^{\infty}(\Omega)$ with respect to the corresponding norm $\|\cdot\|_{X}$. Since

$$
\|\varphi\|_{X} \geqq k_{p}\|\varphi\|_{p+r} \quad\left(\varphi \in C_{0}^{\infty}(\Omega)\right),
$$

one has $X \subset H_{0}^{p+r}(\Omega)$.

We define on $L^{2}(\Omega)$ so-called negative norm $\|\cdot\|_{X}$ by the formula

$$
\|v\|_{X}=\sup _{0 \neq u \in X} \frac{\left|(u, v)_{0}\right|}{\|u\|_{X}} \quad\left(v \in L^{2}(\Omega)\right) .
$$

Let $X^{-}$be the completion of $L^{2}(\Omega)$ with respect to this norm. Take $u \in X$ and $v \in X^{-}$. There is a sequence $\left\{v_{i}\right\} \subset L^{2}(\Omega)$ such that $\left\|v_{i}-v\right\|_{X^{-}} \rightarrow 0$. Since

$$
\left|\left(u, v_{i}\right)_{0}\right| \leqq\|u\|_{X}\left\|v_{i}\right\|_{X^{-}} \quad(i=1,2, \ldots),
$$


we can define the form $(\cdot, \cdot)_{0}$ on $X \times X^{-}$such that

$$
\left|(u, v)_{0}\right| \leqq\|u\|_{X}\|v\|_{X^{-}} \quad\left(u \in X, v \in X^{-}\right) .
$$

Each $v \in L^{2}(\Omega)$ determines a continuous linear functional $(\cdot, v)_{0}$ on $X$. Consequently, there is a continuous linear mapping $A_{0}: L^{2}(\Omega) \rightarrow X$ such that

$$
(u, v)_{0}=\left(u, A_{0} v\right)_{X} \quad\left(u \in X, v \in L^{2}(\Omega)\right) .
$$

For $v \in L^{2}(\Omega)$ one has

$$
\left\|A_{0} v\right\|_{X}=\sup _{0 \neq u \in X} \frac{\left\lfloor\left(u, A_{0} v\right)_{X} \mid\right.}{\|u\|_{X}}=\|v\|_{X^{-}} .
$$

Thus, $A_{0}$ maps a dense subset of $X^{-}$isometrically into $X$. Consequently $A_{0}$ has an isometric extension $A: X^{-} \rightarrow X$. Since $A$ is isometric, the range $A\left(X^{-}\right)$is closed in $X$. If $A\left(X^{-}\right) \neq X$, one can find a non-zero $u \in X$ such that for each $v \in L^{2}(\Omega)$

$$
(u, v)_{0}=(u, A v)_{X}=0 .
$$

This is true only if $u=0$, therefore $A\left(X^{-}\right)=X$. Thus, the mapping $A: X^{-} \rightarrow X$ is an isometric isomorphism, $X^{-}$is a Hilbert space, and

$$
(u, v)_{0}=(u, A v)_{X}=\left(A^{-1} u, v\right)_{X^{-}} \quad\left(u \in X, v \in X^{-}\right) .
$$

This implies that the spaces $X$ and $X^{-}$are dual with respect to the form $(\cdot, \cdot)_{0}$. If $\psi \in C_{0}^{\infty}(\Omega)$, we get

$$
(\varphi, M \psi)_{0}=(\varphi, \psi)_{X}=\left(\varphi, A^{-1} \psi\right)_{0} \quad\left(\varphi \in C_{0}^{\infty}(\Omega)\right),
$$

and therefore $M \psi=A^{-1} \psi$.

Let $D^{\prime}(\Omega)$ be the space of distributions in $\Omega$. An element $u \in X$ determines a distribution

$$
\varphi \rightarrow(\varphi, u)_{0} \quad\left(\varphi \in C_{0}^{\infty}(\Omega)\right) .
$$

We shall identify $u$ with this distribution. Then we get $\mathrm{Y}^{-} \subset D^{\prime}(\Omega)$. If $u, M u \in X^{-}$, we have

$$
(\varphi, M u)_{0}=(M \varphi, u)_{0} \quad\left(\varphi \in C_{0}^{\infty}(\Omega)\right) .
$$

Since $C_{0}^{\infty}(\Omega)$ is dense in $X$ and $A^{-1}: X \rightarrow X^{-}$is an isomorphism, the set $A^{-1}\left(C_{0}^{\infty}(\Omega)\right)=M\left(C_{0}^{\infty}(\Omega)\right)$ is dense in $X^{-}$. Let us define on the domain $D\left(M_{0}\right)=M\left(C_{0}^{\infty}(\Omega)\right)$ an operator $M_{0}: D\left(M_{0}\right) \rightarrow X^{-}$by

$$
M_{0} \psi=M \psi \quad\left(\psi \in D\left(M_{0}\right)\right) \text {. }
$$

Lemma 1. The operator $M_{0}$ is symmetric in $X^{-}$. If $u, M u \in X^{-}$, one has $u \in D\left(M_{0}^{*}\right)$, where $M_{0}^{*}$ is the adjoint of $M_{0}$ in $X^{-}$. 
Proof. For $\psi \in D\left(M_{0}\right)=A^{-1}\left(C_{0}^{\infty}(\Omega)\right)$. one has $\varphi=A \psi \in C_{0}^{\infty}(\Omega)$ and we get by (4)

$$
\begin{gathered}
(\psi, M u)_{X^{-}}=\left(A^{-1} \varphi, M u\right)_{X^{-}}=(\varphi, M u)_{0}=(M \varphi, u)_{0}=(\psi, u)_{0} \\
=\left(A^{-1} \psi, u\right)_{X^{-}}=\left(M_{0} \psi, u\right)_{X_{-}} .
\end{gathered}
$$

This implies that $M_{0}$ is symmetric in $X^{-}, u \in D\left(M_{0}^{*}\right)$ and $M_{0}^{*} u=M u$.

Lemma 2. If $f \in H^{p}(\Omega)$ then $L^{\prime} S^{p} f \in X^{-}$.

Proof. Let $Y$ be the closure of the set $L\left(C_{0}^{\infty}(\Omega)\right)$ in $H^{p}(\Omega)$ and $Y^{\perp}$ the orthogonal complement of $Y$ in $H^{P}(\Omega)$. Then we can decompose $f=v+f_{0}$ where $v \in Y, f_{0} \in Y^{-}$. Since

$$
\left(f_{0}, S^{p} L \varphi\right)_{0}=\left(f_{0}, L q\right)_{p}=0 \quad\left(q \in C_{0}^{\infty}(\Omega)\right) .
$$

one has $L^{\prime} S^{p} f_{0}=0$ and therefore $L^{\prime} S^{p} f=L^{\prime} S^{p} v$. For $\varphi \in C_{0}^{\infty}(\Omega)$ we get

$$
\|L \varphi\|_{p}^{2}=\left(\varphi, L^{\prime} L S^{p} \varphi\right)_{0}=\varphi \|_{x}^{2}
$$

and moreover

$$
\begin{aligned}
\left|\left(L^{\prime} S^{p} v\right)(\varphi)\right| & =\left|v\left(L S^{p} \varphi\right)\right|=\left(\mathrm{v}, L S^{p} \varphi\right)_{0} \mid \\
& =\left|(v, L \varphi)_{p}\right| \leqq v\left\|_{p} L \varphi\right\|_{p}=\left\|v_{p}\right\|_{p}\|\varphi\|_{X} .
\end{aligned}
$$

Thus the distribution $L^{\prime} S^{p} v \in D^{\prime}(\Omega)$ is bounded by the norm $\|\cdot\|_{X}$. Consequently, $L^{\prime} S^{p} f=L^{\prime} S^{p} v \in X^{-}$: since $X^{-}$is dual with $X$ with respect to the form $(\cdot, \cdot)_{0}$.

Theorem 3. Assume that $f \in H^{p}(\Omega)$ and let $u \in X^{-}$be a distribution solution in $\Omega$ of the equation 1

$$
L u=f .
$$

Then $u$ can be decomposed

$$
u=u_{0}-\psi_{1} .
$$

where $u_{0} \in X \subset H_{0}^{p+r}(\Omega)$ and $\vartheta \in X^{-}$satisfies

$$
\left(M^{2}+1\right) \text { it }=0 \text {. }
$$

Proof. According to Lemma 2 one has

$$
M u=L^{\prime} S^{p} L u=L^{\prime} S^{p} f \in \mathrm{X}^{-} .
$$

Thus we get by Lemma $1, u \in D\left(M_{0}^{*}\right)$, where $M_{0}^{*}$ is the adjoint in $X$ of the symmetric operator $M_{0}$. Let $\bar{M}_{0}$ be the closure in $X^{-}$of $M_{0}$. Then $u$ can be decomposed

1 A distribution solution in $\Omega$ of the equation $L u=f$ is defined to be a distribution $u \in D^{\prime}(\Omega)$ such that $(L u)(\varphi):=u\left(L^{\prime} \varphi\right)=f(\varphi)$ for all $\varphi \in C_{0}^{\infty}(\Omega)$. 


$$
u=u_{0}+\vartheta_{1}+\vartheta_{2},
$$

where $u_{0} \in D\left(\bar{M}_{0}\right)$ and the elements $\vartheta_{1}, \vartheta_{2} \in D\left(M_{0}^{*}\right)$ satisfy

$$
M_{0}^{*} \vartheta_{1}=i \vartheta_{1}, \quad M_{0}^{*} \vartheta_{2}=-i \vartheta_{2}
$$

(see e.g. [2], VII.4, Theorem 3, p. 204).

For $\varphi \in C_{0}^{\infty}(\Omega)$ one has $M \varphi \in M\left(C_{0}^{\infty}(\Omega)\right)=D\left(M_{0}\right)=D\left(M_{0}+i I\right)$ and, since $\left(M_{0}+i I\right)^{*} \vartheta_{1}=\left(M_{0}^{*}-i I\right) \vartheta_{1}=0$. we get

$$
\left.\left((M+i) \varphi, \vartheta_{1}\right)_{0}=\left(M_{0}+i I\right) M \varphi \cdot \vartheta_{1}\right)_{X^{-}}=0 .
$$

This implies that $(M-i) \vartheta_{1}=0$. Similarly one has $(M+i) \vartheta_{2}=0$. Writing $\vartheta=\vartheta_{1}+\vartheta_{2}$ we get

$$
\left(M^{2}+1\right) \vartheta=(M+i)(M-i)\left(\vartheta_{1}+\vartheta_{2}\right)=0
$$

Since $u_{0} \in D\left(\bar{M}_{0}\right)$, there exists a sequence $\left\{\psi_{i}\right\} \subset D\left(M_{0}\right)=M\left(C_{0}^{\infty}(\Omega)\right)$ such that

$$
\left\|\psi_{i}-u_{0}\right\|_{X^{-}} \rightarrow 0, \quad\left\|M_{0} \psi_{i}-\bar{M}_{0} u_{0}\right\|_{X^{-}} \rightarrow 0
$$

Because

$$
\left\|\psi_{i}-\psi_{j}\right\|_{X}=\left\|A^{-1}\left(\psi_{i}-\psi_{j}\right)\right\|_{X^{-}}=\left\|M_{0}\left(\psi_{i}-\psi_{j}\right)\right\|_{X^{-}} \rightarrow 0 .
$$

there exists $u_{0}^{\prime} \in X$ such that $\psi_{i} \rightarrow u_{0}^{\prime}$ in $X$. This implies that $\psi_{i} \rightarrow u_{0}^{\prime}$ also in $X^{-}$, therefore $u_{0}=u_{0}^{\prime} \in X$.

Corollary 4. We assume that $L$ is hypoelliptic and $f \in L^{2}(\Omega)$. If $u \in L^{2}(\Omega)$ is a distribution solution of

$$
L u=f,
$$

then there exists a sequence $\left\{\psi_{i}\right\} \subset C^{\infty}(\Omega) \cap L^{2}(\Omega)$ such that in $L^{2}(\Omega)$, $\psi_{i} \rightarrow u$ and $L \psi_{i} \rightarrow f$.

Proof. Let us take $p=0$ and apply Theorem 3. Then we get $u=u_{0}+\vartheta$, where $u_{0} \in X$ and $\vartheta$ satisfies (5) with $M=L^{\prime} L$. Since $L$ is hypoelliptic, $M^{2}+1$ is also hypoelliptic and therefore $\theta \in C^{\infty}(\Omega)$. For $\varphi \in C_{0}^{\infty}(\Omega)$ one has

$$
\|\varphi\|_{X}^{2}=\left(\varphi, L^{\prime} L \varphi\right)_{0}=\|L \varphi\|_{0}^{2} .
$$

Since $u_{0} \in X$ there exists a sequence $\left\{\varphi_{i}\right\} \in C_{0}^{\infty}(\Omega)$ such that $\varphi_{i} \rightarrow u_{0}$ in $X$. Because

$$
\left\|L \varphi_{i}-L \varphi_{j}\right\|_{0}=\left\|\varphi_{i}-\varphi_{j}\right\|_{X} \rightarrow 0 .
$$

there is $v \in L^{2}(\Omega)$ such that $L \varphi_{i} \rightarrow v$ in $L^{2}(\Omega)$. For each $\varphi \in C_{0}^{\infty}(\Omega)$ one has 


$$
\left(L^{\prime} \varphi, \varphi_{i}\right)_{0} \rightarrow\left(L^{\prime} \varphi, u_{0}\right)_{0}=\left(L u_{0}\right)(\varphi)
$$

and on the other hand

$$
\left(L^{\prime} \varphi, \varphi_{i}\right)_{0}=\left(\varphi, L \varphi_{i}\right)_{0} \rightarrow(\varphi, v)_{0}=v(\varphi) .
$$

This implies that $L u_{0}=v$ and therefore $L \varphi_{i} \rightarrow L u_{0}$ in $L^{2}(\Omega)$. Thus we get for $\psi_{i}=p_{i}+\vartheta \in C^{\infty}(\Omega) \cap L^{2}(\Omega), \quad \psi_{i} \rightarrow u_{0}+\vartheta=u \quad$ and $L \psi_{i}=L \varphi_{i}+L \vartheta \rightarrow L u_{0}+L \vartheta=f$ in $L^{2}(\Omega)$.

I am most grateful to the referee, who has read the manuscript and suggested the following theorem, which is simpler and more general than Theorem 3 in the case $r=0$. This theorem also implies Corollary 4 .

Theorem 5. Assume that $f \in H^{P}(\Omega)$ and let $u \in D^{\prime}(\Omega)$ be a distribution solution in $\Omega$ of the equation

$$
L u=f .
$$

Then $u$ can be decomposed

$$
u=u_{0}+\vartheta
$$

where $u_{0} \in H_{0}^{p}(\Omega)$ and $\vartheta \in D^{\prime}(\Omega)$ satisfies

$$
L^{\prime} L S^{p} \vartheta=0 \text {. }
$$

Proof. Lot us define on $C_{0}^{\infty}(\Omega)$ an operator $L_{0}: C_{0}^{\infty}(\Omega) \rightarrow H_{0}^{p}(\Omega)$ by

$$
L_{0} \varphi=L \varphi \quad\left(\varphi \in C_{0}^{\infty}(\Omega)\right) .
$$

It has in $H_{0}^{p}(\Omega)$ a closure $\bar{L}_{0}$ and according to (3) one has

$$
\left\|\bar{L}_{0} v\right\|_{p} \geqq k_{p}\|v\|_{p} \quad\left(v \in D\left(\bar{L}_{0}\right)\right) .
$$

This implies that the range $R\left(\bar{L}_{0}\right)$ is closed in $H_{0}^{p}(\Omega)$ and therefore a closed subspace of $H^{P}(\Omega)$. Thus, the element $f \in H^{P}(\Omega)$ can be decomposed $f=f_{0}+f_{1}$, where $f_{0}$ is contained in $R\left(\vec{L}_{0}\right)$ and $f_{1}$ in the orthogonal complement of $R\left(\bar{L}_{0}\right)$ in $H^{p}(\Omega)$. We can find $u_{0} \in D\left(\bar{L}_{0}\right) \subset H_{0}^{p}(\Omega)$ such that $\bar{L}_{0} u_{0}=f_{0}$. There exists a sequence $\left\{\varphi_{n}\right\} \subset D\left(L_{0}\right)=C_{0}^{\infty}(\Omega)$ such that in $H_{0}^{p}(\Omega)$ one has $\varphi_{n} \rightarrow u_{0}$ and $L \varphi_{n}=L_{0} \varphi_{n} \rightarrow \bar{L}_{0} u_{0}=f_{0}$. Thus, for each $\varphi \in C_{0}^{\infty}(\Omega)$

$$
\left(L^{\prime} \varphi, \varphi_{n}\right)_{0} \rightarrow\left(L^{\prime} \varphi, u_{0}\right)_{0}
$$

and on the other hand

$$
\left(L^{\prime} \varphi, \varphi_{n}\right)_{0}=\left(\varphi, L \varphi_{n}\right)_{0} \rightarrow\left(\varphi, f_{0}\right)_{0} .
$$

Consequently, we have

$$
\left(\varphi, f_{0}\right)_{0}=\left(L^{\prime} \varphi, u_{0}\right)_{0} \quad\left(\varphi \in C_{0}^{\infty}(\Omega)\right) .
$$


We identify $u_{0}$ with the distribution

$$
\varphi \rightarrow\left(\varphi, u_{0}\right)_{0} \quad\left(\varphi \in C_{0}^{\infty}(\Omega)\right)
$$

and write $\vartheta=u-u_{0} \in D^{\prime}(\Omega)$. For each $\psi \in C_{0}^{\infty}(\Omega)$ one has $L S^{p} \psi \in C_{0}^{\infty}(\Omega)$ and using the relation (6) we get

$$
\begin{aligned}
\left(L^{\prime} L S^{p} \vartheta\right)(\psi) & =\left(L^{\prime} L S^{p} u\right)(\psi)-\left(L^{\prime} L S^{p} u_{0}\right)(\psi) \\
& =(L u)\left(L S^{p} \psi\right)-u_{0}\left(L^{\prime} L S^{p} \psi\right) \\
& =\left(L S^{p} \psi, f\right)_{0}-\left(L^{\prime}\left(L S^{p} \psi\right), u_{0}\right)_{0} \\
& =\left(L S^{p} \psi, f\right)_{0}-\left(L S^{p} \psi, f_{0}\right)_{0} \\
& =\left(L S^{p} \psi, f_{1}\right)_{0}=\left(L_{0} \psi, f_{1}\right)_{p}=0
\end{aligned}
$$

since $f_{1}$ is in $H^{p}(\Omega)$ orthogonal to $R\left(\bar{L}_{0}\right) \supset R\left(L_{0}\right)$. Thus we have $L^{\prime} L S^{p} \vartheta=0, \quad u_{0} \in H_{0}^{p}(\Omega)$ and $u=u_{0}+\vartheta$.

Tampere University of Technology

Tampere - Finland

\section{References}

[1] Hönmander, L.: On the theory of general partial differential operators. - Acta Math. 94, 1955, pp. 161-248.

[2] Yosida, K.: Functional analysis. - [Third edition] Die Grundlehren der mathematischen Wissenschaften 123. Springer-Verlag, Berlin / Heidelberg / New York, 1971. 